\title{
REVIEW OF BENOIT CUSHMAN-RoISIN'S INTRODUCTION TO GEOPHYSICAL FLUID DYNAMICS
}

\author{
By Dennis W. Moore
}

$\mathrm{P}_{\mathrm{r}}$ P ductory geophysical fluid dynamics text for "upperlevel undergraduates, graduate students, and researchers in environmental fluid dynamics." His purpose is to provide a suitable introductory text for a broad audience, including not only physicists and mathematicians but also "biologists, atmospheric chemists, and engineers (who wish) to understand the rudiments of climate and ocean dynamics." For those students and others who are unprepared to tackle Pedlosky's Geophysical Fluid Dynamics, Professor Cushman-Roisin's Introduction to Geophysical Fluid Dynamics is an easier place to start. Actually, anyone reading Pedlosky's Geophysical Fluid Dynamics for the first time might find Cushman-Roisin's book to be a useful supplement.

The book is divided into five parts. Part I is called "Fundamentals," with an introductory chapter which explains what geophysical fluid dynamics is, a chapter on the Coriolis force, and one on the governing equations. Each chapter is accompanied by a problem set, some chapters have suggested laboratory demonstrations, and most have biographical information and a picture of a notable geophysical fluid dynamicist. (In chapter 1 there is a sketch of Walsh Cottage, the mecca of geophysical fluid dynamics.)

Part II is on the effects of rotation, with chapters on geostrophy and vorticity, Ekman layers, linear barotropic waves, barotropic instability, and large-scale ocean circulation. Part III, on stratification effects, has chapters on stratification, internal waves, and stratified turbulence. Part IV, on combined effects of rotation and stratification, has chapters on layered models, stratified geostrophic dynamics, upwelling, quasi-geostrophy, baroclinic instability, and fronts, jets and vortices. Finally, Part V has chapters on climate dynamics and equatorial dynamics. There is also an appendix on wave kinematics, and a 10-page reference section listing both books and journal articles.

D.W. Moore, University of Hawaii, School of Ocean and Earth Science and Technology, 1000 Pope Rd., Honolulu, Hawaii 96822 , USA.
This book has many strengths. It covers a broad range of topics and gives a good introduction to them. The overall level at which the material is presented is about right for the intended audience, and the problems are generally good. Most of the illustrations and accompanying explanations are clear and convey the ideas quite well. The historical and biographical notes definitely add interest.

However, I do not agree with everything Cushman-Roisin writes. For example, he says on p. 22, "As far as the author is aware, nobody has yet proposed a clear and convincing physical interpretation for the Coriolis force." I made a modest effort to help Henry Stommel with an introductory book on the Coriolis force, intended for an audience no more sophisticated than the one Cushman-Roisin addresses. He does mention our work ("A very detailed exposition . . ."). I wonder, since the Coriolis force is adventitious, and results solely from the choice of a rotating coordinate system, why one should expect "a convincing physical interpretation"?

A more important concern is the discussion (pp. 115-118) and illustration (Figure 8-3) about "westward intensification." The velocity profile $\mathrm{v}(x)$ in the figure (for example, panel b) ought to have positive curvature everywhere, consistent with equation (8-20). The picture as drawn is inconsistent with the Stommel model, which was both the first and simplest explanation of the westward intensification phenomenon. Furthermore, the discussion should focus on the total vorticity balance in the boundary layer. Interior $V$ is southward, so boundary layer $\mathrm{V}$ must be northward. Northward advection of planetary vorticity in the boundary layer must be balanced by dissipation, proportional to minus the relative vorticity in the Stommel model. So relative vorticity must be negative in the boundary layer, which it can be on the western side but not the eastern side.

Part of the difficulty arises, I think, because perturbation expansions and analysis are really not discussed in any detail, but concepts that depend on perturbation methods as their underlying 
. . . anyone teaching

introductory

geophysical fluid

dynamics should find

this to be a useful

text. basis are invoked in the physical arguments. So on p. 115 for example, we find the phrase "first . . a approximation" in describing equations (813) and (8-14) near the top of the page, and "we note that the $\mathrm{x}$-derivatives are much larger there than in the rest of the domain," near the bottom of the page. Both of these statements depend on unexplained ideas about scaling and ordered perturbation expansions.

On p. 163, the resistance of a fluid to movement is attributed to viscosity. But viscosity inhibits deformation, not motion. On p. 234 , it is claimed that meridional boundaries prevent thermal-wind-type currents in the ocean from encircling the globe. But certainly the Antarctic Circumpolar Current is a counterexample.

I taught a one-semester introductory geophysical fluid dynamics course for four first-year graduate students using a pre-publication copy of this book as the text. We spent a lot of time working problems, which I regard as very important at this level, and did not get through the whole book in a semester. With regard to the problems there is an answer book available that is a definite help to the teacher. Most of the problems are good and a hy- pothetical one about colliding Taylor columns (problem 4-5) is fascinating. Rudolf Kloosterziel and $I$ are still trying to figure out how to implement that one in the lab. (A motorized potter's wheel makes an excellent turntable for laboratory demonstrations.) One problem (2-5) in the Coriolis force chapter is misleading, suggesting the centrifugal force may be neglected when it should not be. One problem in the Ekman layer chapter (5-4) involves a rather laborious calculation which I found uninformative. But these are minor quibbles. A course instructor can pick and choose which problems to use.

Based on an early draft, I encouraged PrenticeHall to publish this book. If it were a journal article, I would probably send it back to the author for minor revisions. Perhaps there will be a second edition. In any case, anyone teaching introductory geophysical fluid dynamics should find this to be a useful text.

\section{Acknowledgments}

I am indebted to Eric Firing, who has also taught from this book, for helpful discussions as I prepared this review. 